\title{
Research of Ateb-Gabor filter in biometric protection systems
}

\author{
Mariya Nazarkevych \\ Department of Information Technology Publishing \\ Lviv Polytechnic National University, \\ mariia.a.nazarkevych@lpnu.ua \\ Nataliya Lotoshynska \\ Department of Information Technology Publishing \\ Lviv Polytechnic National University, Lviv, Ukraine, \\ natlot@ukr.net
}

\author{
Volodymyr Buriachok \\ Department of Information and cybersecurity \\ Borys Grinchenko Kyiv University, Ukraine \\ v.buriachok@kubg.edu.ua
}

\section{Sergiy Dmytryk}

Department of Information Technology Publishing Lviv Polytechnic National University, Lviv, Ukraine, sergiy.dmytruk@gmail.com

\begin{abstract}
Biometric recognition systems require the development of new technologies and need improvement. A new filter of Ateb-Gabor has been investigated. The filter is based on the use of a combination of Gabor filter and periodic Atebfunctions. This combination allows us to provide flexibility for control by choosing two parameters $m$ and $n$, which is provided by the mathematical apparatus of the Ateb functions. Filtration was performed on the example of biometric images. It has been shown that Ateb-Gabor filtration gives better filtration results than ordinary Gabor filter. Experimental research has been carried out.
\end{abstract} filtering

Keywords - Ateb-Gabor filter, Ateb-functions, image

\section{INTRODUCTION}

Today, modern systems of identification and protection of information continue to develop the most dynamically in the security industry. Particular attention is attracted to the biometric means of information protection, due to their high reliability and significant reduction in prices recently achieved. Using these means allows to raise automated systems of diverse destination on a fundamentally new level of quality. This is due to the prospects of using biometrics, the versatility of biometric characteristics and the development of information technologies [1,2].

Many publications are devoted to the problems of use of biometric technologies for the information protection in both open and closed literary sources, in particular. The importance of scientific achievements and contribution to the theory and practice of information security of these scientists cannot be overestimated [3].

Thus, Jain and Lakhmi C. consider [4] that recognition systems by the retina or the iris or fingerprints are inconvenient in practice because of the stringent requirements for the order of scanning person's biometric characteristics. According to some authors [5], the main reasons for the popularity of fingerprint recognition techniques are: the success of their use in various forensic or governmental applications; criminals often leave fingerprints at the scene of a crime; the availability of large hereditary databases; affordability of compact and cheap fingerprint devices. Many issues in the field of biometric protection remain unsolved. One question is related to the recognition and comparison accuracy. This article is devoted to issue of increasing the recognition accuracy by developing a new method of filtration

Methods of biometric authentication of computer users can be divided into two large groups: static and dynamic [4]. Classification of biometric authentication methods is presented in Fig. 1.

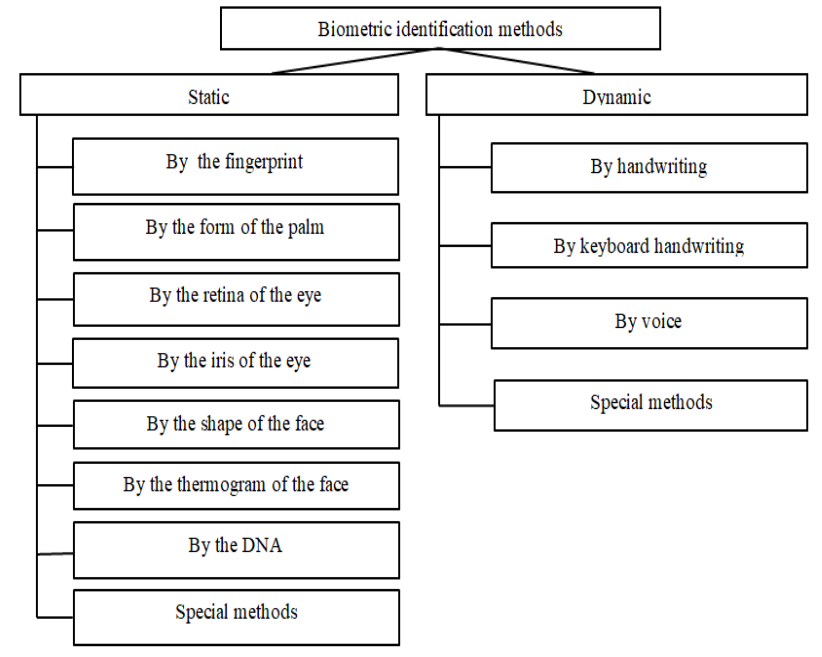

Fig. 1. Classification of methods of biometric authentication of users

The analysis of dynamic authentication methods has shown that, despite their drawbacks (high probability of authentication errors and false alarms of the security system), they are still needed in small institutions, enterprises and organizations due to their simplicity and accessibility [2]. 
From the point of view of the prevalence of biometric techniques "three large biometrics" are distinguished: fingerprint identification, face geometry and eye iris. According to some authors, fingerprint identification systems occupy more than half of the biometric technologies market, systems based on the face geometry recognition technology have $13-18 \%$, and the system based on the eye iris identification have 6-9\%. And to a lesser extent, in the information security systems, such techniques as identification by the eye retina, by DNA, by the image of the hand, by the pattern of the palm veins or finger of the hand, by the face thermogram, by the forms of the ear bowl, by the smell, by the voice, by signature, by keyboard handwriting and by analyzing of bioelectric activity of the brain [3].

The most reliable among practically implemented methods is the method of scanning the retina of the eye. Therefore, it is used in access control systems for particularly sensitive objects. Due to the low level of distribution of such systems, the probability of breakthrough attempts realization is low. But the disadvantage of systems using this method is their high cost.

The reliability of the system is achieved by combining several biometric recognition methods. Each system built separately does not ensure $100 \%$ protection, so it is advisable to set up combined biometric systems (fingerprint and voice recognition, eye iris and fingerprint recognition, voice and three-dimensional image, etc.).

It should be noted that the situation in the biometric systems market changes very quickly. New technologies constantly appear that are more reliable and often cheaper.

\section{ANALYSIS OF IMAGE FILTRATION METHODS}

At the present stage, images have become an integral part of human life, as well as an important part of many technology branches. The scope of their use is continuously expanding. Computerized procedures are used to facilitate perception of images in industry, medicine and security systems. An important point is obtaining images in modern systems for identification and protection of information.

If the image was obtained using digitization, there is usually noise on it. Noise distorts the digital image. Therefore, the processing and image quality enhancement in order to improve their visual perception of the person as well as to solve problems related to machine perception of images is an important area of modern work and needs constant improvement and improvement of the methods by which these tasks can be performed.

\section{FORMULATION OF THE PROBLEM}

The aim of the work is to study the new methods of filtering images in the biometric protection systems and to prove their effectiveness. In particular, to investigate the properties of filtration by Ateb-Gabor. Experimental data is given.

\section{IMAGE FILTRATION METHODS}

The obtained image is observed with instruments can be mathematically written as:

$$
\mathrm{v}(\mathrm{i})=u(i)+\mathrm{n}(\mathrm{i})
$$

where $v(i)$ is the observed value, $u(i)$ is the actual value that needs to be restored from $v(i), n(i)$ is the noise deviation.

The clearing noise method from image $u$ can be defined as Dh :

$$
u=D h u+n(D h, u)
$$

where $\mathrm{h}$ is the filtration parameter, $D h u$ is image cleared of noise and $n(D h, u)$ is noise determined by this method.

At the moment, it's not enough just to smooth $u$ and get a cleared image. More advanced methods not only smooth the image, but also try to restore the lost information $\mathrm{n}(\mathrm{Dh}, \mathrm{u})$, if it is needed, in the image it is often necessary to maintain clarity and detail while the noise needs to be blurred.

There are many ways to improve image quality. The following methods can be used:

1. Local filtration methods, including:

- Gaussian model of smoothing, where smoothing is measured by the Dirichlet integral $\int|D u|^{2}$;

- filter of Yaroslavl boundaries and Susan filter;

- bilateral filter.

2. Methods based on partial differential equation, including:

- model of anisotropic filtration;

- model of the complete variation of Rudin-Osher-Fatemi;

3. Filters in the frequency domain including:

- local adaptive filters in the transformation areas;

- hard and soft thresholding;

- Zhou-Wang wavelet full variation.

4. Nonlocal averaging algorithm (NL-means).

Each of the listed filters modifies the image and reduces or blurs the noise.

However, for biometric images, the above filters are not widely used. In biometric images, it is necessary to highlight the contours and make a comparison with the bank of originals. Gabor filtration is used for this purpose.

\section{ATEB-GABOR FILTRATION}

Gabor's filter is a linear electronic filter whose impulse transient characteristic is determined in the form of a harmonic function multiplied by the Gaussian function [6]. For filtering images, in particular biometric images, it is necessary to apply a two-dimensional Gabor filter that can filter in twodimensional space [7].

We suggested to use the Ateb-Gabor filter. The Ateb-Gabor filter is a filter whose impulse transition characteristic is determined in the form of a harmonic function in the form of the Ateb-function multiplied by the Gaussian function. Such application will greatly increase the filtering results. This will yield significantly more filtering results, as there are two new $\mathrm{m}, \mathrm{n}$ parameters that can be changed [8].

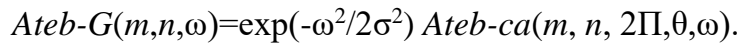


where $\sigma$ is standard deviation of the Gaussian nucleus, defines the amplitude of the function, $\theta$ is frequency of oscillation which is defined as $\theta=1 / T$, where $T(m, n)$ is the period of Atebca $(m, n, 2 \Pi, \theta, \omega)$.

Graphical representation of frequency characteristics is shown in Fig. 2, 3, 4. Fig. 2 shows the normal Gabor filter, which is displayed with the parameters of the Ateb-function [9] $m$ and $n$ of the unit level. Ten figures show a change of $\omega$ from 0.1 to 1.0. As we can see from Fig. 2, changing the $\omega$ value, changes the filtration area scale. Fig. 3 shows the frequency characteristics of the Ateb-Gabor filter with parameters $m=n$ $=3$. Looking on the frequency characteristics, we can see that the images are significantly different from the ordinary Gabor filter. Fig. 4 shows the frequency characteristics of the AtebGabor filter with parameters $m=n=5$. As we can see from the frequency characteristics, the images look more saturated and more contrasting than the Gabor filter.

Figures 2, 3, 4 show the results of filtration of the AtebGabor. As a result, we get a bank of filters that differ in scale, which is actually shown in Figures 2, 3, 4. The same bank of figures can be obtained by changing the angle of inclination $\theta$ from formula (3). Each biometric image is unique and requires its selection of parameters. Below are some good experimental results. Searching for optimal parameters for a specific image is the theme of another research. As a result of filtration, the images are modified by converting the brightness of the corresponding pixel, which is shown in the filter bank with the corresponding pixel of the biometric image, thus contrast in certain areas of the image increases.

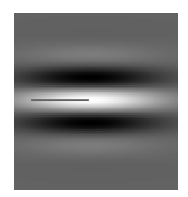

$\mathrm{m}=1, \mathrm{n}=1, \omega=0.1$

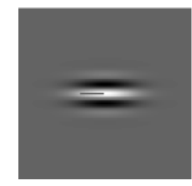

$\mathrm{m}=1, \mathrm{n}=1, \omega=0.4$

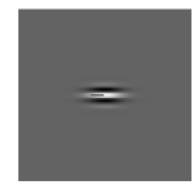

$\mathrm{m}=1, \mathrm{n}=1, \omega=0.7$

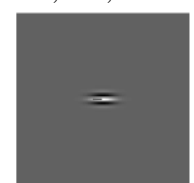

$\mathrm{m}=1, \mathrm{n}=1, \omega=1.0$

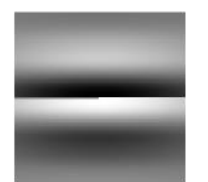

$\mathrm{m}=3, \mathrm{n}=3, \omega=0$
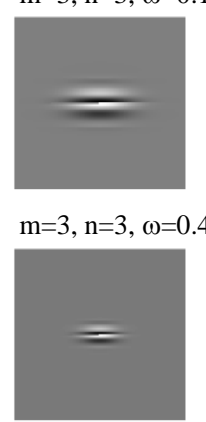

$\mathrm{m}=3, \mathrm{n}=3, \omega=0.7$

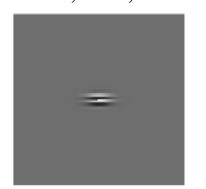

$\mathrm{m}=3, \mathrm{n}=3, \omega=1.0$ $\mathrm{m}=3, \mathrm{n}=3, \omega=0.4$

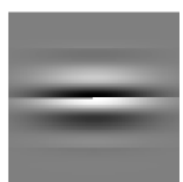

$\mathrm{m}=3, \mathrm{n}=3, \omega=0.2$

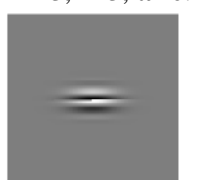

$\mathrm{m}=3, \mathrm{n}=3, \omega=0.5$

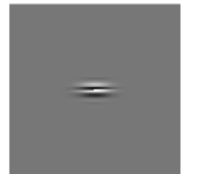

$\mathrm{m}=3, \mathrm{n}=3, \omega=0.8$

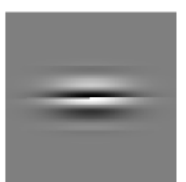

$\mathrm{m}=3, \mathrm{n}=3, \omega=0.3$

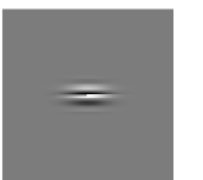

$\mathrm{m}=3, \mathrm{n}=3, \omega=0.6$

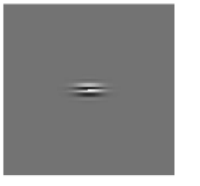

$\mathrm{m}=3, \mathrm{n}=3, \omega=0.9$
Fig. 3. Graphic representation of frequency two-dimensional Ateb-Gabor for $\mathrm{m}=3, \mathrm{n}=3$

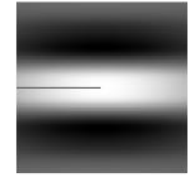

$m=5 n=5 \omega=0.1$

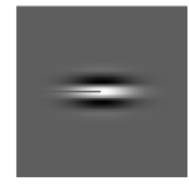

$\mathrm{m}=3 \mathrm{n}=3 \omega=0.4$

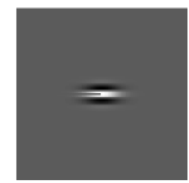

$\mathrm{m}=3 \mathrm{n}=3 \omega=0.7$

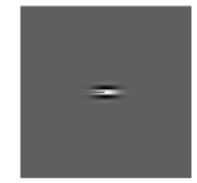

$\mathrm{m}=5 \mathrm{n}=5 \omega=1$

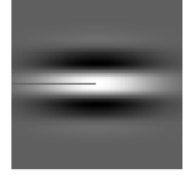

$\mathrm{m}=5 \mathrm{n}=5 \omega=0.2$

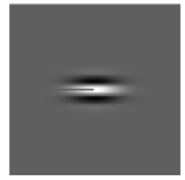

$\mathrm{m}=3 \mathrm{n}=3 \omega=0.5$

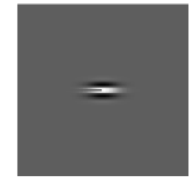

$\mathrm{m}=5 \mathrm{n}=5 \omega=0.8$

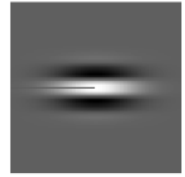

$m=5 n=5 \omega=0.3$

$m=3 n=3 \omega=0.6$

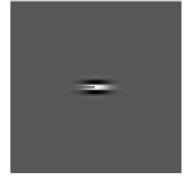

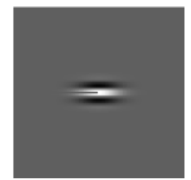

$m=5 n=5 \omega=0.9$

Fig. 4. Graphic representation of frequency two-dimensional Ateb-Gabor for $\mathrm{m}=5, \mathrm{n}=5$

Fig. 2. Graphic representation of frequency two-dimensional Ateb-Gabor for $m=1, n=1$ 


\section{EXPERIMENTAL RESEARCH}

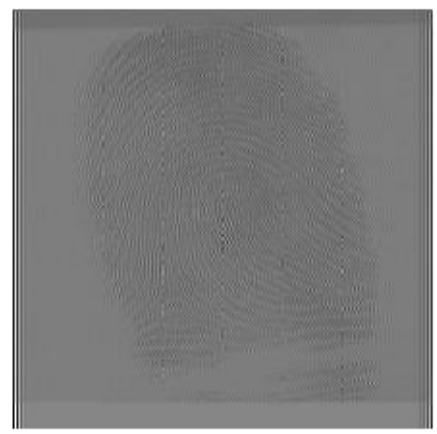

Fig. 5. Filtered image of Ateb-Gabor with parameters $m=1, n=1, \omega=0.1$

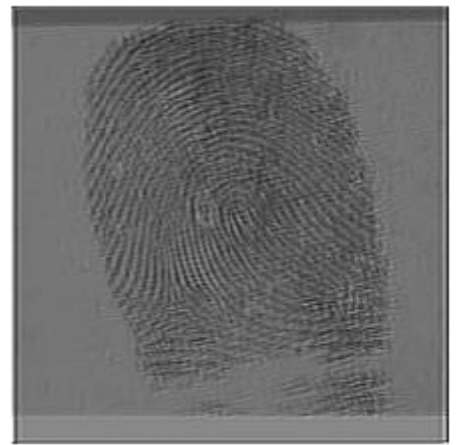

Fig. 6. Filtered image of Ateb-Gabor with parameters $m=3, n=3, \omega=0.1$

Examples of filtered image are shown in Fig. 5 and 6. The example of the filtered image of the Ateb-Gabor with parameters $\mathrm{m}=3, \mathrm{n}=3$ is much better than the usual Gabor, shown in Fig. 5 . Therefore, we see the expedient introduction of a new method of filtering, which provides good quality indicators.

There are many filters that allow you to modify the image. Some of the most famous are given in Section 4 of this article. The Gabor filter makes it possible to modify the image without changing the overall contrast and brightness. The proposed filter Ateb-Gabor can even more flexibly modify the biometric image through two independent parameters $\mathrm{m}$, $\mathrm{n}$. In this research, these parameters are equal to each other. But you can achieve better filtering results when they are different. The degree of image enhancement in this article has not been evaluated, but such studies exist. The evaluation was carried out through a correlation comparison of the signal-noise of the input and the filtered image. The larger the parameters $m, n$, the biometric image becomes blurry and less interconnected.

The filtration program is written in Python and does not use third-party libraries. When writing the program, approaches to image processing were proposed, which are proposed in $[10,11]$.

\section{CONCLUSION}

Images that fall into the biometric recognition system have low quality due to noise and other external influences (lighting, glitches). The new Ateb-Gabor filtration method is considered, which reduces noise and interference effects and creates an effective filtration method.
The Ateb-Gabor filter has been developed, which extends the functions of control of an ordinary Gabor filter by generalizing trigonometric functions. This allows you to develop a new image processing method.

The developed filtering method has many properties, as it is based on the Ateb-function theory.

Experimental studies of the comparison of the new AtebGabor filter and ordinary Gabor have been carried out. The comparison is made using correlation methods.

In this study, a bank of two-dimensional Ateb-gauge filtered in the frequency domain was developed with parameters $m=3$, $\mathrm{n}=3$ and $\mathrm{m}=5, \mathrm{n}=5$, and a comparison of the two-dimensional Ateb-gauge with parameters $\mathrm{m}=1, \mathrm{n}=1$, which is identical filter of two-dimensional Gabor. In addition, new software was developed at Pyton, which allowed for the experimental results presented in section 6 .

Experimental results show an improvement in the level of filtration in Fig. 6, when the Ateb-Gabor was filtered with parameters $\mathrm{m}=3, \mathrm{n}=3, \mathrm{w}=0.1$, compared to the usual twodimensional Gabor (Fig. 5) $\mathrm{m}=1, \mathrm{n}=1, \mathrm{w}=0.1$. Therefore, this development is relevant and requires further research.

\section{REFERENCES}

[1] Tuyls, P., Akkermans, A. H., Kevenaar, T. A., Schrijen, G. J., Bazen, A. M., \& Veldhuis, R. N. (2005, July). Practical biometric authentication with template protection. In International Conference on Audio-and Video-Based Biometric Person Authentication (pp. 436-446). Springer, Berlin, Heidelberg.

[2] N. D. T. Tieu, H. H. Nguyen, H. Q. Nguyen-Son, J. Yamagishi and I. Echizen, "An approach for gait anonymization using deep learning," 2017 IEEE Workshop on Information Forensics and Security (WIFS), Rennes, 2017, pp. 1-6.

[3] W. D. Herrera, O. L. León, W. C. Londoño and J. A. Vargas, "Implementation of a control and biometric safety of the vascular network of the dorsal side of the hand through digital processing of images," 2017 IEEE 3rd Colombian Conference on Automatic Control (CCAC), Cartagena, 2017, pp. 1-6.

[4] Jain, Lakhmi C., et al., eds. Intelligent biometric techniques in fingerprint and face recognition. Vol. 10. CRC press, 1999.

[5] Jain, Anil K., Jianjiang Feng, and Karthik Nandakumar. "Fingerprint matching." Computer 43.2 (2010).

[6] Jones, Judson P., and Larry A. Palmer. "An evaluation of the twodimensional Gabor filter model of simple receptive fields in cat striate cortex." Journal of neurophysiology 58.6 (1987): 1233-1258.

[7] Yang, Jianwei, et al. "A modified Gabor filter design method for fingerprint image enhancement." Pattern Recognition Letters24.12 (2003): 1805-1817.

[8] Nazarkevych, Mariya, Roman Oliarnyk, and Sergiy Dmytruk. "An images filtration using the Ateb-Gabor method." Computer Sciences and Information Technologies (CSIT), 2017 12th International Scientific and Technical Conference on. Vol. 1. IEEE, 2017.

[9] Nazarkevych, M., Oliiarnyk, R., Nazarkevych, H., Kramarenko, O., \& Onyshschenko, I. (2016, August). The method of encryption based on Ateb-functions. In Data Stream Mining \& Processing (DSMP), IEEE First International Conference on (pp. 129-133). IEEE.

[10] O. Riznik, I. Yurchak, E. Vdovenko and A. Korchagina, "Model of stegosystem images on the basis of pseudonoise codes," 2010 Proceedings of VIth International Conference on Perspective Technologies and Methods in MEMS Design, Lviv, 2010, pp. 51-52.

[11] O. Riznyk, I. Yurchak and O. Povshuk, "Synthesis of optimal recoverysystems in distributed computing using ideal ring bundles," 2016 XIIInternational Conference on Perspective Technologies and Methods in MEMSDesign (MEMSTECH), Lviv, 2016, pp. 220-222. doi: 10.1109/MEMSTECH.2016.7507545. 